\title{
Clinical Utility of a Biomarker to Detect Contrast-Induced Acute Kidney Injury during Percutaneous Cardiovascular Procedures
}

\author{
John Peabody a, b, c David Paculdo ${ }^{a}$ Czarlota Valdenor ${ }^{a}$ Peter A. McCullough ${ }^{d}$ \\ Eisei Noiri ${ }^{,}$f Takeshi Sugayag, h Jeffrey R. Dahlen ${ }^{\mathrm{i}}$

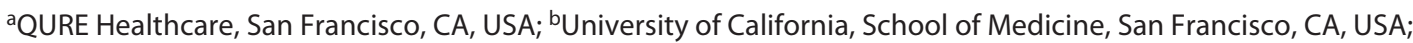 \\ 'University of California, Fielding School of Public Health, Los Angeles, CA, USA; ${ }^{\mathrm{d}}$ Texas Christian University and the

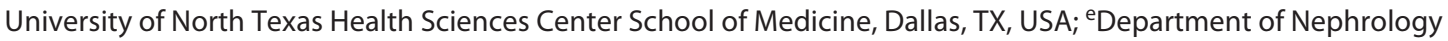 \\ and Endocrinology, The University of Tokyo Hospital, Tokyo, Japan; ${ }^{\mathrm{f}}$ National Center Biobank Network, National \\ Center for Global Health and Medicine, Tokyo, Japan; 9Timewell Medical, Tokyo, Japan; 'ht. Marianna University \\ School of Medicine, Kawasaki, Japan; 'Hikari Dx, Inc., San Diego, CA, USA
}

\section{Keywords}

Contrast-induced nephropathy · Cardiac catheterization ·

Coronary intervention · Quality of care · Vascular

intervention

\begin{abstract}
Introduction: Contrast-induced acute kidney injury (Cl-AKI) is a major clinical complication of percutaneous cardiovascular procedures requiring iodinated contrast. Despite its relative frequency, practicing physicians are unlikely to identify or treat this condition. Methods: In a 2-round clinical trial of simulated patients, we examined the clinical utility of a urine-based assay that measures liver-type fatty acid-binding protein (L-FABP), a novel marker of Cl-AKI. We sought to determine if interventional cardiologists' ability to diagnose and treat potential $\mathrm{Cl}-\mathrm{AKI}$ improved using the biomarker assay for 3 different patient types: pre-procedure, peri-procedure, and post-procedure patients. Results: 154 participating cardiologists were randomly divided into either control or intervention. At baseline, we found no difference in the demographics or how they identified and treated potential complications of AKI, with both groups providing less than
\end{abstract}

karger@karger.com www.karger.com/crm

Karger $\frac{1}{6}$
(C) 2022 The Author(s)

Published by S. Karger AG, Basel

This is an Open Access article licensed under the Creative Commons Attribution-NonCommercial-4.0 International License (CC BY-NC) (http://www.karger.com/Services/OpenAccessLicense), applicable to the online version of the article only. Usage and distribution for commercial purposes requires written permission. half the necessary care to their patients ( $46.4 \%$ for control vs. $47.6 \%$ for intervention, $p=0.250$ ). The introduction of L-FABP into patient care resulted in a statistically significant improvement of $4.6 \%(p=0.001)$. Compared to controls, physicians receiving L-FABP results were 2.9 times more likely to correctly identify their patients' risk for AKI $(95 \% \mathrm{Cl} 2.1$ 4.0) and were more than twice as likely to treat for AKI by providing volume expansion and withholding nephrotoxic medications. We found the greatest clinical utility in the preprocedure and peri-procedure settings but limited value in the post-procedure setting. Conclusion: This study suggests L-FABP as a clinical marker for assessing the risk of potential $\mathrm{Cl}-\mathrm{AKI}$, has clinical utility, and can lead to more accurate diagnosis and treatment.

(c) 2022 The Author(s). Published by S. Karger AG, Basel

\section{Introduction}

Percutaneous cardiovascular interventions, which now number $>1.6$ million occurrences annually, pose a unique challenge to the cardiologist [1]. High-quality care requires balancing between evaluating and treating 
the underlying diseases and avoiding complications from the diagnostic procedure [2], which is done in patients with many risk factors [3].

With the large volume of percutaneous cardiovascular contrast procedures, contrast-induced acute kidney injury (CI-AKI) has emerged as a major clinical problem [4]. CI-AKI is now the third most common cause of AKI in the hospital setting after hypovolemia and nephrotoxic medications [5]. It is recognized as a frequent complication of percutaneous cardiovascular procedures, occurring between 7 and $18 \%$ of patients undergoing percutaneous coronary intervention (PCI) [6-8]. CI-AKI adds an estimated incremental cost of USD 10,667 per case [9]. Other studies consistently show the occurrence of AKI is associated with an increase in both morbidity and mortality [3], leading to more heart attacks, longer in-hospital stays, a more complicated hospitalization course, and higher in-hospital morbidity and mortality [5].

AKI prediction and prevention is an important healthcare priority because current therapeutic options are limited once AKI develops [10]. The pre-procedure setting is the best time to identify at-risk patients by properly assessing factors leading to developing CI-AKI: pre-existing chronic kidney disease (CKD), older age, diabetes, heart failure, use of nephrotoxic drugs, and volume of contrast [11]. CI-AKI can be mitigated in the peri- and post-procedural settings after it occurs [11]. While debate continues over how effective prevention and risk mitigation are, volume expansion is an effective intervention to reduce the risk for CI-AKI whether it occurs in the pre-, peri-, or post-procedural setting and, hence, is the strong recommendation included in guidelines [12, 13].

While treatments may be limited, a foundational shortcoming faced by interventional cardiologists is that there is currently no reliable way to predict CI-AKI development. In a recent article, we documented cardiologists were able to diagnose renal insufficiency appropriately and make the corresponding correct assessment of CIAKI risk 59.2\% of the time [14]. Preventive strategies through hydration, removal of nephrotoxic drugs, the use of iso-osmotic contract material, or delaying the procedure cannot be fully employed unless diagnostic risk is better assessed. In this same report, there was significant variation in the method and frequency at which cardiologists implemented strategies directed toward lowering CI-AKI risk, with only $15.8 \%$ providing the necessary prophylactic care. We also explored whether the risk factors were identified in the pre-procedure, peri-procedure, and post-procedure settings, and found that they identi- fied the risk of developing CI-AKI $29.9 \%$ of the time in the pre-procedure patients, versus $46.5 \%$ for peri-procedure patients, versus $45.9 \%$ in the post-procedure patients [14].

Serum creatinine is the most common functional biomarker used to test for renal impairment. Creatinine, however, lacks the sensitivity to measure early declines in the glomerular filtration rate to detect early AKI: as much as a $50 \%$ loss of renal function may have occurred prior to an appreciable rise in serum creatinine [15]. Serum creatinine also lacks specificity in differentiating from other sources of renal injury, such as prerenal azotemia and other causes of pre-existing CKD. This injury, if detected by better biomarkers, creates the potential to more accurately identify patients at risk, intervene earlier with prophylactic measures, more efficiently monitor these patients for late outcomes, and produce better outcomes. One such biomarker is liver-type fatty acid-binding protein (L-FABP).

$\mathrm{L}-\mathrm{FABP}$ when measured in urine is a sensitive and specific indicator of renal ischemia. Urinary levels of the L-FABP have been shown to increase earlier than serum creatinine in patients who develop CI-AKI, and L-FABP levels can increase as early as $4 \mathrm{~h}$ after IV contrast exposure in the peri-procedural period [16]. Other studies have shown that L-FABP is not only a good diagnostic predictor of CI-AKI but it also has prognostic significance with higher urinary baseline levels being associated with eventual need for dialysis [17].

We conducted a prospective, randomized in silico study using Clinical Performance and Value $\left(\mathrm{CPV}^{\circledR}\right)$ vignettes to determine if introducing L-FABP as a biomarker increased the identification of patients at risk for CI-AKI and led to greater prevention and better treatment. CPV vignettes are well-known, validated simulated patient cases used to determine if diagnostic tests meet the criteria of clinical utility for payor coverage and reimbursement $[18,19]$. The CPVs involve a physician evaluating and providing care for a simulated patient by having the physician respond to open-ended queries divided into 5 domains of care: (1) taking a history, (2) performing a physical examination, (3) ordering diagnostic workup, (4) making a diagnosis, and (5) determining the treatment and follow-up care [20, 21]. By construct, the use of simulated patients adjusts for casemix variation because all providers care for the same set of potential use cases for the new diagnostic test. Clinical utility is assessed by evaluating the effect of the L-FABP test results on the physician's evaluation and treatment decisions.
Peabody/Paculdo/Valdenor/McCullough/ Noiri/Sugaya/Dahlen 


\section{Materials and Methods}

\section{Overview}

The QUINCE (QURE Utility of INterventional Cardiology Evaluation) study is a randomized controlled trial conducted between March and April 2020. It aims to measure the clinical utility among interventional cardiologists in the USA of the RENISCHEM $^{\circledR}$ L-FABP POC test used to detect AKI. We measured the participating physicians' clinical performance in caring for 9 different simulated patients at risk for CI-AKI before and after the introduction of the L-FABP test.

\section{Ethics}

This study was conducted in accordance with ethical standards, approved by the Advarra Institutional Review Board, Columbia, MD, USA, and listed in clinicaltrials.gov (NCT04266834). Online informed consent was obtained from all participants.

\section{Physician Selection}

We invited 298 interventional cardiologists to join the study from a nationally representative list of over 6,000 practitioners. They were deemed eligible to join the study if the following inclusion criteria were met: (1) board-certification in cardiology, (2) at least $20 \mathrm{~h}$ averaged per week of clinical and patient care duties over the last 6 months, and (3) at least $8 \mathrm{~h}$ per month spent performing invasive or interventional cardiology procedures in a catheterization lab. Overall, 169 of the 298 invited participants were deemed eligible to be in the study. However, 15 did not complete both rounds of the study, leaving 154 participants who were ultimately included. We found no differences between those eligible participants who completed the study and those who did not $(p>0.05$ for all). All participants completed a health provider questionnaire consisting of questions on demographics, training, and practice environment as part of data collection.

\section{Intervention}

154 interventional cardiologists were randomly assigned to either the intervention group (77) or the control group (77). After baseline data collection (round 1) was completed, participants in the intervention group received educational material on the L-FABP test. The educational material included an orientation to the test results through a one-page fact sheet, an educational deck with key publications and information on the product, and a sample package insert with example test results.

\section{Measurement Using $C P V^{\circledR}$ Vignettes}

Nine CPV cases were prepared and grouped into 3 patient case types: (1) outpatients who require urgent interventional care (preprocedure patients), (2) admitted patients who just underwent an interventional procedure (peri-procedure), and (3) post-discharge patients with late-onset CI-AKI (post-procedure). Upon completion of the vignettes, an overall score and a domain score for diagnosis plus treatment (including preventive measures) were calculated using the explicit, predetermined evidence-based criteria. The scorers were blinded to the participant's assignment and identity.

\section{Administration of the $C P V^{\circledR}$ Vignettes}

We administered 2 rounds of vignettes to the participants. The participants were randomly assigned to complete 3 cases for the first, or baseline, round. During the second, or post-intervention, round, participants were given 3 additional CPV cases. Only the intervention group had access to simulated L-FABP test results. Cases are given in a random order to obviate any possible ordering effect.

Overall, each of the 154 physician participants cared for $6 \mathrm{CPV}$ patients for a total of $924 \mathrm{CPV}$ simulated patients, split evenly between the 3 case types (pre-, peri-, and post-procedure). From the physician responses provided, we compared their overall ability to provide evidence-based clinical care for their patients, paying special attention to diagnostic accuracy of AKI risk and correctly outlining of treatment for these patients: the choice and volume of contrast material for the pre-procedure patients, repeat creatinine testing for the peri- and post-procedure patients, and, for all patients, initial renal function assessment, volume expansion, and stopping/withholding nephrotoxic medications.

\section{Outcomes}

The primary outcome was a change in the overall CPV scores for CI-AKI, diagnostic accuracy, AKI risk stratification, and use of preventive and/or therapeutic interventions. From previous studies, we know that a clinically significant change of $3 \%-5 \%$ in overall scores means there is a change in real-world outcomes [22].

\section{Analyses}

Summary statistics were determined for all variables. Numerical variables were summarized through mean and standard deviation. For categorical outcomes, we used Fisher's exact test and logistic regression for multivariate modeling. For continuous outcomes, $t$-test and linear regression were performed. CPV domain and treatment scores were adjusted using multiple linear and logit regression models to control potential confounders. All analyses were performed in Stata 14.2.

\section{Results}

The 154 board-certified physicians were randomly divided, 77 into the intervention group and 77 into the control group (Table 1). We found little to no significant difference in the characteristics between the 2 groups: around $90 \%$ of enrolled cardiologists being male, nearly two-thirds working in urban locales, and about $40 \%$ working in private practice. The only statistically significant difference was that the intervention group was slightly more likely to be employed by their practice (97.4\%) versus the control group (87.0\%) $(p=0.016)$.

At baseline, the overall clinical care scores were nearly identical between the 2 groups $(46.4 \%$ vs. $47.6 \%, p=$ 0.250 ) (Table 2). We found these similarities held true for all 3 case types, in the diagnosis domain, in making the primary cardiovascular diagnosis, the secondary diagnoses, or in their assessments of AKI risk where there were no differences ( $p>0.05$ for all), except that intervention doctors diagnosed CKD at a higher rate than the controls ( $56.1 \%$ vs. $45.0 \%, p=0.024)$ at baseline. In the treatment 
Table 1. Comparison of provider characteristics by study arm

\begin{tabular}{|c|c|c|c|}
\hline & Control & Intervention & $p$ value \\
\hline$N$ & 77 & 77 & - \\
\hline Male, \% & 89.6 & 92.2 & 0.575 \\
\hline Age & $51.4 \pm 9.6$ & $50.6 \pm 9.2$ & 0.614 \\
\hline \multicolumn{4}{|l|}{ Setting, \% } \\
\hline Urban & 61.0 & 62.3 & \multirow{3}{*}{0.173} \\
\hline Suburban & 36.4 & 28.6 & \\
\hline Rural & 2.6 & 9.1 & \\
\hline \multicolumn{4}{|l|}{ Region, \% } \\
\hline Northeast & 26.0 & 26.0 & \multirow{4}{*}{0.986} \\
\hline Midwest & 20.8 & 22.1 & \\
\hline South & 33.8 & 31.2 & \\
\hline West & 19.5 & 20.8 & \\
\hline \multicolumn{4}{|l|}{ Practice type, \% } \\
\hline Private practice, solo & 2.6 & 2.6 & \multirow{5}{*}{0.985} \\
\hline Private practice, single specialty & 27.3 & 24.7 & \\
\hline Private practice, multispecialty & 9.1 & 9.1 & \\
\hline Community health system & 20.8 & 24.7 & \\
\hline Academic & 40.3 & 39.0 & \\
\hline Time outpatient, \% & $46 \% \pm 18$ & $48 \% \pm 20$ & 0.506 \\
\hline Time interventional procedures, $\%$ & $26 \% \pm 22$ & $26 \% \pm 21$ & 0.896 \\
\hline Employed by practice & 87.0 & 97.4 & 0.016 \\
\hline \multicolumn{4}{|l|}{ Payer, \% } \\
\hline Medicare & 42.2 & 40.5 & 0.590 \\
\hline Medicaid & 17.4 & 16.5 & 0.714 \\
\hline Commercial & 34.9 & 34.5 & 0.881 \\
\hline Self & 3.7 & 4.1 & 0.634 \\
\hline Other & 1.8 & 4.4 & 0.137 \\
\hline \multicolumn{4}{|l|}{ CMS participation } \\
\hline MIPS & 27.3 & 27.3 & 1.000 \\
\hline $\mathrm{BPCl}$ & 11.7 & 18.2 & 0.258 \\
\hline Other & 7.8 & 2.6 & 0.146 \\
\hline Do not know & 32.5 & 26.0 & 0.376 \\
\hline Other & 25.7 & 28.6 & 0.584 \\
\hline
\end{tabular}

domain, we saw no difference between the 2 groups, with both arms providing the same amount of necessary care ( $26.6 \%$ for controls vs. $26.4 \%$ for intervention, $p=0.880$ ) as defined in 3 areas: in initial treatment, follow-up, and preventive care.

\section{Overall Management}

Across all settings, we found cardiologists in the intervention group using L-FABP improved their care in the pre-post analysis. Access to L-FABP results improved overall performance $+4.6 \%$ more than control, which is significant both statistically $(p=0.001)$ and clinically (Table 2). Diagnostically, intervention doctors were 2.9 times more likely to correctly identify their patients as being at risk for AKI (95\% CI 2.1-4.0) than controls (Table 3). In percentage terms, the intervention group improved from $33.3 \%$ to $54.1 \%(p<0.001)$ compared to the control group, which only improved from $25.1 \%$ to $33.3 \%$ ( $p=0.052$ ). The difference-in-difference measure of clinical utility between the 2 groups was $12.6 \%(p<0.001)$.

Similarly, intervention cardiologists were more likely to appropriately provide volume expansion (OR 2.2, 95\% CI 1.2-4.0) and to appropriately withhold nephrotoxic medications (OR 2.1, 95\% CI 1.4-3.1) across all 3 case types. Interestingly, there was no difference between the 2 groups in performing or in relying upon repeat in-hospital creatinine testing (OR 1.1, 95\% 0.5-2.3) or keeping patients in the hospital for further evaluation among the peri- and post-procedure patients (OR 1.0, 95\% CI 0.51.9). We found, too, that better risk evaluation with L-FABP led to better overall treatment in the 3 groups. When AKI risk was assessed correctly, the intervention group was more likely to provide volume expansion (OR 2.0, 95\% CI 1.2-3.9), withhold nephrotoxic medications 
Table 2. Overall CPV score and diagnosis + treatment

Table 3. Odds ratio of Cl-AKI-related diagnosis and treatment, intervention versus control

\begin{tabular}{llll}
\hline CPV domain & \multicolumn{2}{l}{ Round } & p value \\
\cline { 2 - 3 } & 1 & 2 & \\
\hline Overall & & & \\
Control, \% & $46.4 \pm 9.9$ & $44.0 \pm 9.7$ & 0.008 \\
Intervention, \% & $47.6 \pm 11.6$ & $49.8 \pm 10.7$ & 0.036 \\
$p$ value & 0.250 & $<0.001$ & 0.001 \\
\hline Diagnosis + treatment & & & \\
Control, \% & $36.1 \pm 15.2$ & $35.0 \pm 13.4$ & 0.394 \\
Intervention, \% & $36.6 \pm 14.8$ & $37.5 \pm 13.1$ & 0.456 \\
$p$ value & 0.743 & 0.038 & 0.279 \\
\hline Primary cardiac diagnosis & & & \\
Control, \% & 84.9 & 84.9 & 1.000 \\
Intervention, \% & 85.3 & 83.6 & 0.608 \\
$p$ value & 0.896 & 0.702 & 0.718 \\
\hline Cardiac risk stratification & & & \\
Control, \% & 46.6 & 48.6 & 0.728 \\
Intervention, \% & 47.8 & 47.2 & 0.911 \\
$p$ value & 0.829 & 0.809 & 0.746 \\
\hline CKD diagnosis & & & 0.191 \\
Control, \% & 45.0 & 54.2 & 0.707 \\
\hline Intervention, \% & 56.1 & 62.4 & \\
$p$ value & 0.024 & 0.093 & \\
\hline
\end{tabular}

CKD, chronic kidney disease; CPV, Clinical Performance and Value.

\begin{tabular}{llr}
\hline & OR & $95 \% \mathrm{Cl}$ \\
\hline Risk of Cl-AKI & 2.9 & $2.1-4.0$ \\
Appropriate volume expansion & 2.2 & $1.2-4.0$ \\
$\begin{array}{l}\text { Appropriate withholding of nephrotoxic medications } \\
\text { In-hospital creatinine testing }\end{array}$ & 2.1 & $1.4-3.1$ \\
$\begin{array}{l}\text { Extend in-hospital admission for peri- and post-procedure patients } \\
\quad \text { for further evaluation }\end{array}$ & 1.1 & $0.5-2.3$ \\
When AKI correctly assessed & 1.0 & $0.5-1.9$ \\
$\quad$ Provide volume expansion & 2.0 & $1.2-3.9$ \\
$\quad$ Withhold nephrotoxic medications & 2.0 & $1.4-2.7$ \\
$\quad$ In-hospital creatinine testing for post-procedure patients & 3.0 & $2.1-4.5$ \\
\hline
\end{tabular}

$\mathrm{Cl}-\mathrm{AKI}$, contrast-induced acute kidney injury.
(OR 2.0, 95\% CI 1.4-2.7), and repeat in-hospital creatinine levels for the post-procedure patients (OR 3.0, 95\% CI 2.1-4.5).

\section{Lowering AKI Risk in the Pre-Procedure Patients}

Next, we looked specifically at patients being evaluated prior to the angiographic study. We found that the overall provision of care improved $+4.4 \%$ with L-FABP testing compared to the control group, and this improvement was statistically significant $(p=0.047)$. In the setting of an elevated L-FABP test, which indicated an increased likelihood of AKI, the intervention cardiologists dramatically improved their assessment of AKI risk from $22.1 \%$ in the first round to $45.5 \%$ in the second round. When we com- 
pared this to the control group (20.8\% at baseline vs. $16.9 \%$ in round $2, p=0.536$ ) the pre- versus post-difference-indifference between the 2 groups was $+27.3 \%(p=0.015)$.

Not surprisingly, providers who identified a patient as at risk for CI-AKI were more likely to use volume expansion (O.R. 2.2, $p<0.001)$. The addition, however, of a positive L-FABP test further increased the likelihood of hydrating the patient (O.R. 2.2. $p=0.011)$. Intervention doctors were also significantly more likely to stop or withhold nephrotoxic medications prior to the procedure, with a difference-in-difference of $+9.1 \%(p=$ $0.003)$. There was no difference, however, between the 2 groups in matching the use of higher cost iso-osmolar contrast agents to patients who were at risk. Several observations that did not reach statistical significance showed trends toward improved utilization of contrast media, such as using an iso-osmolar contrast agent in the intervention group $(+12.7 \%, p=0.364)$, using an isoosmolar contrast agent when AKI risk was correctly identified $(+5.6 \%, p=0.725)$, using a less contrast agent in the intervention group $(+8.3 \%, p=0.493)$, and using a less contrast agent when AKI risk was correctly identified $(+13.6 \%, p=0.411)$. When combining either the use of an iso-osmolar agent or a lower volume of a contrast agent, the results similarly demonstrated a trend that did not reach statistical significance (intervention vs. control $+17.6 \%, p=0.190$; identifying correct AKI risk $+18.5 \%, p=0.155)$.

\section{Managing AKI in the Peri- and Post-Procedure \\ Patients}

As found in the pre-procedure patients, over 2 rounds, introduction of L-FABP in the peri- and post-procedure patients significantly improved the overall, evidencebased care by $+4.7 \%$, compared to controls $(p=0.007)$. The improvement was similar in the peri-procedure or post-procedure setting $(+4.5 \%[p=0.076]$ and $+5.0 \%$ $[p=0.036])$, respectively. However, in the setting of an elevated L-FABP test, the intervention group caring for peri-procedural patients increased their ability to assess AKI risk from $33.8 \%$ to $63.6 \%$, with a between-group difference of $14.3 \%(p<0.001)$. Similarly, the difference-indifference (logit) model showed that intervention doctors using L-FABP were significantly more likely to diagnose $\mathrm{CKD}$ in the peri-procedure patients $(+14.3 \%, p=0.036)$, but this was not the case for the post-procedure patients $(+0.0 \%, p=0.401)$.

Next, we examined whether intervention group physicians were more likely to correctly treat CI-AKI with volume expansion and found a much larger and significant improvement for the peri-procedure cases (differencein-difference $24.7 \%, p=0.028)$ than for the post-procedure cases (difference-in-difference 10.4\%, $p=0.308$ ). With regard to withholding nephrotoxic medications such as metformin, ACE inhibitors, and ARBs in the CPV cases, there was a larger effect size for the peri-procedure cases, with a difference-in-difference of $+17.0 \%$ ( $p=$ 0.017 ), and no effect in the post-procedure cases (difference-in-difference of $0.0 \%, p=0.983$ ).

Predischarge monitoring of creatinine is typically performed for the evaluation of late-onset CI-AKI. In the peri- and post-procedure patients, intervention group physicians were 2.4 times more likely (95\% CI 0.8-7.4) to correctly monitor their peri-procedure patients, but only 0.7 times as likely $(95 \% \mathrm{CI} 0.2-2.2)$ for their post-procedure patients.

Choice of TAVR versus SAVR. Two cases involved patients requiring aortic valve replacement. The intervention group physicians, who had access to (elevated) L-FABP results, were more likely to recommend surgical aortic valve replacement instead of transcatheter aortic valvular replacement, which carries a greater risk of CI-AKI (odds ratio 5.0, 95\% CI 0.2-195.1), and overall, the difference-in-difference is $9.4 \%$, but this was not significant ( $p=0.391$ ). One of the cases involved a valve replacement patient with elevated creatinine but a normal L-FABP result. For this case, the intervention physicians were more likely to correctly proceed with the TAVR procedure (OR 9.0, 95\% CI 0.8-101.2), or in percentage terms, there was a difference-in-difference of $+27.1 \%$ that approached statistical significance $(p=0.075)$.

\section{Creatinine Monitoring and Discharge from Hospital}

The likelihood that creatinine would be checked prior to discharge was evaluated, and intervention group physcians using L-FABP were $15.6 \%(p=0.040)$ more likely to reassess the creatinine in the peri-procedure patient cases, but there was no difference pre-post in the post-procedure cases. Additionally, physicians who did not stratify AKI correctly in both intervention and control groups were only one-third as likely to order predischarge creatinine (OR 0.3, $p<0.001$ ).

In the combined post-procedure patients, we found intervention physicians trended towards improved discharge disposition of their patients, defined as either keeping the patient in the hospital or discharging them, with greater improvement in the discharge of peri-procedure patients (difference-in-difference $+25.4 \%, p=0.070$ ) and no improvement in the post-procedure patients (difference-in-difference $+5.8 \%, p=0.770$ ).
Cardiorenal Med 2022;12:11-19 DOI: $10.1159 / 000520820$
Peabody/Paculdo/Valdenor/McCullough/ Noiri/Sugaya/Dahlen 


\section{Discussion}

The purpose of the study was to determine the clinical utility of a new L-FABP measurement to increase the ability of interventional cardiologists to identify the risk of CI-AKI in their patients undergoing interventional studies and optimize their treatment. With the steady growth of percutaneous angiographic procedures, to current estimates of over 1.6 million procedures annually, there has been a corresponding increase in CI-AKI, which we estimate to be in the neighborhood of 192,000 (12\%) cases per year [1]. Clinically, it is clear that a rise in creatinine, which most providers rely upon to identify renal injury, happens too late to avoid, delay, or mitigate the risks of AKI. In our earlier work, we documented that interventional cardiologist correctly determined a patient's risk for CI-AKI only $29.2 \%$ of the time [14].

The pathophysiology of CI-AKI is complex and may often be multifactorial, with both ischemic and inflammatory events. All formulations of contrast media have shown to be cytotoxic in vitro. In addition to damaging the surrounding endothelium, the contrast media have a cytotoxic effect on the tubules of the nephron. These changes result in sustained vasoconstriction, leading to a decreased glomerular filtration rate [9]. This injury has the potential to result in long-lasting consequences, implying the possibility of a longitudinal relationship between CI-AKI and recurrent renal injury. There exists a practice gap in the follow-up of patients with CI-AKI in which the association between CI-AKI and adverse events after hospital discharge remains less well defined, with the risk of recurrent renal injury understudied and the timing of these events still unknown. In a recent analysis from the National Cardiovascular Data Registry (NCDR) using the Acute Kidney Injury Network (AKIN) definition of $\mathrm{AKI}$, it was found that AKI remains a common complication after PCI, occurring in $8.8 \%$ of those patients with periprocedural assessment of renal function. Furthermore, in-hospital AKI is associated with significant morbidity and mortality after hospital discharge. This analysis demonstrates an association between inhospital AKI after PCI and higher rates of post-discharge mortality, MI, bleeding-related hospitalization, and recurrence of renal insufficiency at 1 year when compared with patients without in-hospital AKI [23].

One striking finding is the enormous variation in how well interventional cardiologists mitigated risk and acted on prognostic implications of CI-AKI. Evidence-based practice, in our study, ranged from $3.9 \%$ to $66.7 \%$. We found that the introduction of L-FABP measurements led to a modest but significant $+4.6 \%$ improvement in overall quality of care. Diagnostic scores improved even more by $12.6 \%$ with the availability of L-FABP measurements, while volume expansion and withholding nephrotoxic medications were more than twice as likely (2.3 and 2.1 times, respectively) and implementing close monitoring of renal function across all 3 case types was 3 times more likely. These data show the potential of L-FABP as an early and reliable biomarker for assessing the patient's risk of developing of CI-AKI.

The use of L-FABP guided the use of lower dye loads but not the potentially more cost-effective iso-osmotic contrast agents. Several meta-analyses showed no difference between low-osmolar contrast media and iso-osmolar contrast media in terms of renal safety [24], which might also explain why cardiologists did not change these practices. However, it is equally plausible that the lack of significance reflects that in many catheterization labs, interventionalists do not have a choice in the dye type they use. When asked to choose between a higher dye load contrast procedure (TAVR) versus a surgical alternative (SAVR), interventional cardiologists who had the L-FABP indicated they would do the TAVR when the L-FABP was not increased and would avoid contrast in those patients where the L-FABP was high and indicated a greater risk for CI-AKI (trend to improvement).

We evaluated 3 different settings where L-FABP could be used, and we found L-FABP has the greatest utility in pre-procedure risk mitigation and peri-procedural treatment settings, but only limited value in the post-procedure setting. When used pre-procedurally, L-FABP increases the ability to accurately assess the risk of CI-AKI by $27.3 \%(p=0.015)$, and cardiologists were more likely to order volume expansion (O.R. 2.1), avoid nephrotoxic meds (O.R. 3.0), and anticipate CI-AKI by ordering more frequent renal function monitoring (O.R. 1.1).

In the peri-procedural setting, L-FABP led to higher rates of volume expansion $(+24.7 \%, p=0.028)$, avoidance of nephrotoxic medications $(+17 \%, p=0.017)$, and closer monitoring of renal function $(+15.6 \%, p=0.040)$. The improvement in intravascular volume expansion in this setting is important because it addresses alterations in renal ischemia and direct tubular toxicity that are considered to be the primary factors in the pathogenesis of contrast media-associated nephropathy. While L-FABP's impact was less in the peri- and post-procedure settings, the finding that L-FABP led to an improvement in volume expansion independent of correct risk assessment may indicate L-FABP is potentially perceived as being more reliable than creatinine. It is in the peri-procedure setting 
where more efficient biomarkers such as L-FABP are especially needed to identify CI-AKI, as creatinine levels are slow to manifest a noticeable change in the critical first 48-72 $\mathrm{h}$ after the contrast exposure.

In the post-procedural setting, we found the availability of L-FABP did not significantly improve renal function monitoring or recognition of CI-AKI. While some studies have shown that L-FABP has been shown to have late prognostic significance [25], this did not translate into improved treatment among our study participants.

Our findings are corroborated by other studies that also suggest L-FABP may be useful as a predictive marker of CI-AKI [26]. This study, using specific case scenarios, expands on this to show that L-FABP may be particularly useful in the pre-procedure setting and that L-FABP testing led to better risk assessment and wider use of hydration and withholding nephrotoxic medication in patients at risk.

This study reflects on an important, more general observation. We were looking for better biomarkers of CIAKI because there is and has been an inconsistent and inadequate assessment of risk for patients undergoing a percutaneous procedure, and an even more modest translation of correct risk assessment into meaningful clinical and therapeutic decisions [27]. Although we found that introduction of L-FABP measurements was beneficial in many ways, some of these effects were modest. The difficulties in diagnosing CI-AKI and the limitations facing cardiologists are pervasive in today's practice and have given rise to a sense of "CI-AKI inevitability," which in turn leads to less risk assessment and fewer interventions. This study may be particularly helpful because it shows there may be additional clinical utility that will come from greater awareness. We report herein that L-FABP improved the identification of at-risk patients from $33 \%$ to $54 \%$ across all cases $(p<0.001)$, and that it does lead to prophylactic and therapeutic hydration, which improved by $13.8 \%$ and $24.7 \%$, and did so in the pre-angiographic and the peri-angiographic setting $(p<0.001)$. Importantly, these practice changes are known to lead to better health outcomes. We believe that greater awareness will lead to more and newer interventions that will further reduce morbidity from strokes, myocardial infarctions, and end-stage renal disease requiring dialysis that are clearly linked to CI-AKI [28]. Accordingly, the sensitivity and specificity of L-FABP as an indicator of renal ischemia suggest that it may be useful for continual monitoring of ischemic burden on the kidneys in the setting of existing or new experimental threapies to either prevent or treat AKI.

\section{Limitations}

Our study is limited by several factors. While we made a careful effort to present cases that are commonly encountered in practice, the 9 cases used in this study only cover some of the clinical scenarios and not the full scope of situations where L-FABP could be used in interventional cardiology. Some of the trends such as dye load, renal function monitoring, and timing of discharge were not statistically significant due to underpowering for these analyses. This study also did not collect actual patient data, and although CPV simulations have been validated against actual practice in numerous studies [22,29], future research can address this. Because several trends that did not reach statistical significance were observed, it is also possible that the study was underpowered to identify statistically significant improvements in the selection and use of contrast media. Lastly, since only interventional cardiologists were included, the exclusion of other care team members such as primary care physicians and nephrologists may not have revealed other care patterns, especially in the post-procedural setting where these providers exert a more prominent role.

These results suggest that L-FABP would be useful for assessing a patient's risk for developing CI-AKI and would facilitate better physician decisions for the prevention and treatment of CI-AKI. L-FABP measurements in clinical practice can lead to more accurate diagnosis and monitoring, quicker results/responses, and heightened awareness of a serious and potentially preventable complication facing interventional cardiologists.

\section{Statement of Ethics}

This study was conducted in accordance with ethical standards, approved by the Advarra Institutional Review Board, Columbia, MD, USA, and listed in clinicaltrials.gov (NCT04266834). Online informed consent was obtained from all participants.

\section{Conflict of Interest Statement}

CPVs ${ }^{\circledR}$, QURE Healthcare's proprietary simulated case tool, were used to collect data and score the responses. Dr. Sugaya is an employee of Timewell Medical, the study funder. Otherwise, there are no additional disclosures to report.

\section{Funding Sources}

This study was funded by Timewell Medical, Tokyo, Japan.
18

Cardiorenal Med 2022;12:11-19 DOI: 10.1159/000520820
Peabody/Paculdo/Valdenor/McCullough/ Noiri/Sugaya/Dahlen 


\section{Author Contributions}

J.P., P.A.M., and J.R.D. contributed to the conception; J.P., P.A.M., E.N., and T.S. contributed to the design; C.V., T.S., and J.R.D. contributed to supervision; C.V. and D.P. contributed to data collection; J.P. and D.P. contributed to data analysis; J.P. and D.P. contributed to manuscript drafting. All authors contributed to critical review and the final draft.

\section{Data Availability Statement}

The data supporting the findings of this study are available from the corresponding author, upon reasonable request.

\section{References}

1 Virani SS, Alonso A, Benjamin EJ, Bittencourt MS, Callaway CW, Carson AP, et al. American heart association council on epidemiology and prevention statistics committee and stroke statistics subcommittee. Heart Disease and stroke statistics-2020 update: a report from the american heart association. Circulation. 2020 Mar 3;141(9):e139-e596.

2 Klein LW, Anderson HV, Rao SV. Performance metrics to improve quality in contemporary percutaneous coronary intervention practice. JAMA Cardiol. August 2020;5(8): 859-60.

3 Azzalini L, Candilio L, McCullough PA, Colombo A. Current risk of contrast-induced acute kidney injury after coronary angiography and intervention: a reappraisal of the literature. Can J Cardiol. 2017 Oct;33(10):12258.

4 Ronco F, Tarantini G, McCullough PA. Contrast induced acute kidney injury in interventional cardiology: an update and key guidance for clinicians. Rev Cardiovasc Med. 2020 Mar 30;21(1):9-23.

5 Atanda AC, Olafiranye O. Contrast-induced acute kidney injury in interventional cardiology: emerging evidence and unifying mechanisms of protection by remote ischemic conditioning. Cardiovasc Revasc Med. 2017; 18(7):549-53.

6 Tsai TT, Patel UD, Chang TI, Kennedy KF, Masoudi FA, Matheny ME, et al. Contemporary incidence, predictors, and outcomes of acute kidney injury in patients undergoing percutaneous coronary interventions: insights from the NCDR Cath-PCI registry. JACC Cardiovasc Interv. 2014 Jan;7(1):1-9.

7 Azzalini L, Vilca LM, Lombardo F, Poletti E, Laricchia A, Beneduce A, et al. Incidence of contrast-induced acute kidney injury in a large cohort of all-comers undergoing percutaneous coronary intervention: Comparison of five contrast media. Int J Cardiol. $2018 \mathrm{Dec}$ 15;273:69-73.

8 Silvain J, Nguyen LS, Spagnoli V, Kerneis M, Guedeney P, Vignolles N, et al. Contrast-induced acute kidney injury and mortality in ST elevation myocardial infarction treated with primary percutaneous coronary intervention. Heart. 2018 May;104(9):767-72.

9 Amin AP, Bach RG, Caruso ML, Kennedy KF, Spertus JA. Association of variation in contrast volume with acute kidney injury in patients undergoing percutaneous coronary in- tervention. JAMA Cardiol. 2017;2(9):100712.

10 Meersch M, Schmidt C, Hoffmeier A, Van Aken H, Wempe C, Gerss J, et al. Prevention of cardiac surgery-associated AKI by implementing the KDIGO guidelines in high risk patients identified by biomarkers: the PrevAKI randomized controlled trial. Intensive Care Med. 2017;43(11):1551-61.

11 Morcos R, Kucharik M, Bansal P, Al Taii H, Manam R, Casale J, et al. Contrast-induced acute kidney injury: review and practical update. Clin Med Insights Cardiol. 2019;13: 1179546819878680.

12 Faggioni M, Mehran R. Preventing contrastinduced renal failure: a guide. Interv Cardiol. 2016;11(2):98-104.

13 McCullough PA, Choi JP, Feghali GA, Schussler JM, Stoler RM, Vallabahn RC, et al. Contrast-induced acute kidney injury. J Am Coll Cardiol. 2016;68(13):1465-73.

14 Valdenor C, McCullogh PA, Paculdo D, Acelajado MC, Dahlen JR, Noiri E, et al. Measuring the variation in the prevention and treatment of CI-AKI among interventional cardiologists. Curr Prob Cardiol. 2021;46(9): 100851.

15 He Y, Deng Y, Zhuang K, Li S, Xi J, Chen J. Predictive value of cystatin $\mathrm{C}$ and neutrophil gelatinase-associated lipocalin in contrast-induced nephropathy: a meta-analysis. PLoS One. 2020;15(4):e0230934.

16 Bachorzewska-Gajewska H, Poniatowski B, Dobrzycki S. NGAL (neutrophil gelatinaseassociated lipocalin) and L-FABP after percutaneous coronary interventions due to unstable angina in patients with normal serum creatinine. Adv Med Sci. 2009;54(2): 221-4.

17 Andreucci M, Faga T, Riccio E, Sabbatini M, Pisani A, Michael A. The potential use of biomarkers in predicting contrast-induced acute kidney injury. Int J Nephrol Renovasc Dis. 2016;9:205-21.

18 Peabody JW, Strand V, Shimkhada R, Lee R, Chernoff D. Impact of rheumatoid arthritis disease activity test on clinical practice. PLoS One. May 2013;85:e63215-7.

19 Peabody J, Rahim A, Wilcox B, McGehee C, Estigarribia E, Paculdo D, et al. Clinical utility of a blood-based protein assay on diagnostic colonoscopy referrals for elevated-risk colorectal cancer patients in primary care. Am J Clin Oncol. 2019.
20 Peabody J, Paculdo D, Acelajado MC, Burgon T, Dahlen JR. Finding the clinical utility of 1,5-anhydroglucitol among primary care practitioners. J Clin Transl Endocrinol. 2020;20:100224.

21 Peabody J, Paculdo D, Tamondong-Lachica D, Cabaluna IT, Gunn J. Randomized trial on the clinical utility of a novel biomarker panel to identify treatable determinants of chronic pain. Diagnostics. 2020;10(8):513.

22 Burgon TB, Cox-Chapman J, Czarnecki C, Kropp R, Guerriere R, Paculdo D, et al. Engaging primary care providers to reduce unwanted clinical variation and support ACO cost and quality goals: a unique provider-payer collaboration. Popul Health Manag. 2019; 22(4):321-9.

23 Valle JA, McCoy LA, Maddox TM, Rumsfeld JS, Ho PM, Casserly IP, et al. Longitudinal risk of adverse events in patients with acute kidney injury after percutaneous coronary intervention: insights from the national cardiovascular data registry. Circ Cardiovasc Interv. 2017; 10(4): 0004439.

24 Reed M, Meier P, Tamhane UU, Welch KB, Moscucci M, Gurm HS. The relative renal safety of iodixanol compared with low-osmolar contrast media: a meta-analysis of randomized controlled trials. JACC Cardiovasc Interv. 2009 Jul;2(7):645-54

25 Ferguson MA, Vaidya VS, Waikar SS, Collings FB, Sunderland KE, Gioules CJ, et al. Urinary liver-type fatty acid-binding protein predicts adverse outcomes in acute kidney injury. Kidney Int. 2010;77(8):708-14.

26 Nakamura T, Sugaya T, Node K, Ueda Y, Koide $\mathrm{H}$. Urinary excretion of liver-type fatty acid-binding protein in contrast medium-induced nephropathy. Am J Kidney Dis. 2006 Mar;47(3):439-44.

27 Silver SA, Shah PM, Chertow GM, Harel S, Wald R, Harel Z. Risk prediction models for contrast induced nephropathy: systematic review. BMJ. 2015;351:h4395.

28 Weisbord SD, Palevsky PM. Contrast-induced acute kidney injury: short- and longterm implications. Semin Nephrol. 2011; 31(3):300-9.

29 Yurso M, Box B, Burgon T, Hauck L, Tagg K, Clem $\mathrm{K}$, et al. Reducing unneeded clinical variation in sepsis and heart failure care to improve outcomes and reduce cost: a collaborative engagement with hospitalists in a multistate system. J Hosp Med. 2019 Jun 19;14(9): E1-E6. 